\title{
ESTIMATION OF THE RANGE OF ELASTIC INTERACTION DUE TO GRAIN MISORIENTATION
}

\author{
H. KIEWEL ${ }^{1}$, H. J. BUNGE ${ }^{2}$ and L. FRITSCHE ${ }^{1}$ \\ ${ }^{I}$ Institute of Theoretical Physics $B$, \\ ${ }^{2}$ Department of Physical Metallurgy, Technical University Clausthal, Germany
}

(Received 30 January 1996)

\begin{abstract}
The discontinuities of the elastic constants across grain boundaries in polycrystalline aggregates can be considered as sources of "compatibility" strains in the adjacent crystals under the action of an external stress. This effect was studied theoretically using the cluster model of Kiewel and Fritsche. In order to distinguish the contributions of individual grains, the orientation of only one grain was changed whereas all others in the cluster were kept constant. The orientation dependent part of the strain field thus allows to estimate the range over which the influence of an individual grain reaches. The results shows that this part decreases faster than $1 / r^{3}$ with the distance from the centre of the considered grain. It has virtually vanished at two or three times the grain diameter.

The magnitude of the compatibility strains depends on grain shape. It was found higher in a cluster of cubic grains than in clusters consisting of dodecahedral or cubo-octahedral grains.

The compatibility strains vary systematically when the orientation of the considered grain is changed continuously. The influence of even small misorientations $<5^{\circ}$ is distinctly visible.

The results show that grain shape and orientation correlation must be taken into consideration additional to the texture in order to obtain narrower bounds for the effective elastic constants than those by Voigt and Reuss. They show, however, also that orientation correlations of higher than next-nearest neighbours have virtually no influence.
\end{abstract}

KEY WORDS: Elastic properties, elastic range, misorientation, cluster method.

\section{INTRODUCTION}

Elastic properties of a polycrystalline material are not completely determined by the elastic constants of the constituting single crystals and their Orientation Distribution Function (ODF), since these quantities do not include any interaction between different grains. The polycrystal data which one obtains this way are the well-known values of Voigt (1910) and Reuss (1929) which are only rough estimates of the effective properties. Their most important meaning is that they give upper and lower bounds of the actual effective data (Hill, 1952). To obtain more realistic results additional parameters of the microstructure have to be taken into consideration (Zeller and Dederichs (1973); Kröner (1977)). For example, the grain shape has a significant influence on the effective elastic moduli (see Kiewel, Bunge and Fritsche (1995)).

Another important property of the microstructure consists in the orientation correlation between different grains. As a quantitative measure for the correlation of neighbouring grains the MisOrientation Distribution Function (MODF) may be used. If there is no orientation correlation the MODF of a material with random texture is constant and equal to unity. For this case, Hashin and Shtrikman (1962a, b) obtain bounds which are much narrower than those of Voigt and Reuss. In order to study the influence of 
orientation correlation on the elastic properties of polycrystals we make use of the cluster method by Kiewel and Fritsche (1994) which includes the grain interaction explicitly into the calculation.

\section{METHOD}

In the cluster method as described by Kiewel and Fritsche (1994) an aggregate of single crystals is embedded in a homogeneous medium that possesses approximately the effective elastic constants of the cluster. The embedding minimizes effects which would appear in the case of a free surface. The cluster is subjected to a homogeneous deformation at its entire surface, i.e. we give a combination of displacement and stress at the boundary of the cluster. By solving this boundary value problem under the constraint that displacement and stress are continuous at the grain boundaries the deformation field inside the material is determined.

An instructive quantity describing the deformation behaviour of the investigated material is the relative change of the specific volume

$$
\frac{\delta V(r)}{V_{0}}=\operatorname{Tr}\left(\epsilon(r)-\epsilon_{0}\right)
$$

where $\epsilon(r)$ denotes the strain tensor at the location $r$ in the material, and the constant strain $\epsilon_{0}$ results from the given homogeneous deformation at the surface of the cluster. Thereby the origin of the cluster is situated in its centre of gravity. Forming the trace of the strain tensor is a way of averaging the elastic field and may therefore serve as a criterion to characterize the degree of deformation. Furthermore, we consider the average of this quantity over a sphere with radius $r$ with respect to the chosen origin

$$
\chi(r)=\frac{1}{4 \pi} \int_{0}^{2 \pi} \int_{0}^{\pi} \frac{\delta V(r, \vartheta, \varphi)}{V_{0}} \sin \vartheta d \vartheta d \varphi
$$

where we have introduced spherical coordinates $(r, \vartheta, \varphi)$.

\section{RESULTS AN DISCUSSION}

To obtain representative results we have carried out investigations for three different types of clusters. The first type consists of 365 cubes, the second one of 201 WignerSeitz cells of a face centred cubic (fcc) lattice and the third one of 181 Wigner-Seitz cells of a body centred cubic (bcc) lattice. The individual grains are arranged in shells, i.e. our construction scheme starts with one grain in the origin which is then surrounded by nearest neighbours, next nearest neighbours and so on. For clarity we have confined our investigations to macroscopically isotropic copper, i.e. random orientation distribution and cubic crystal symmetry. Thereto the orientations of individual grains are determined by a random generator. All clusters are subjected to the same uniaxial strain in $\mathrm{X}$-direction at their entire surface.

Results for the deformation field inside clusters consisting of cubes are displayed in Figure 1. For each cluster we have taken the same set of random orientations except for the grain containing the origin of the cluster, which always has different orientations, 


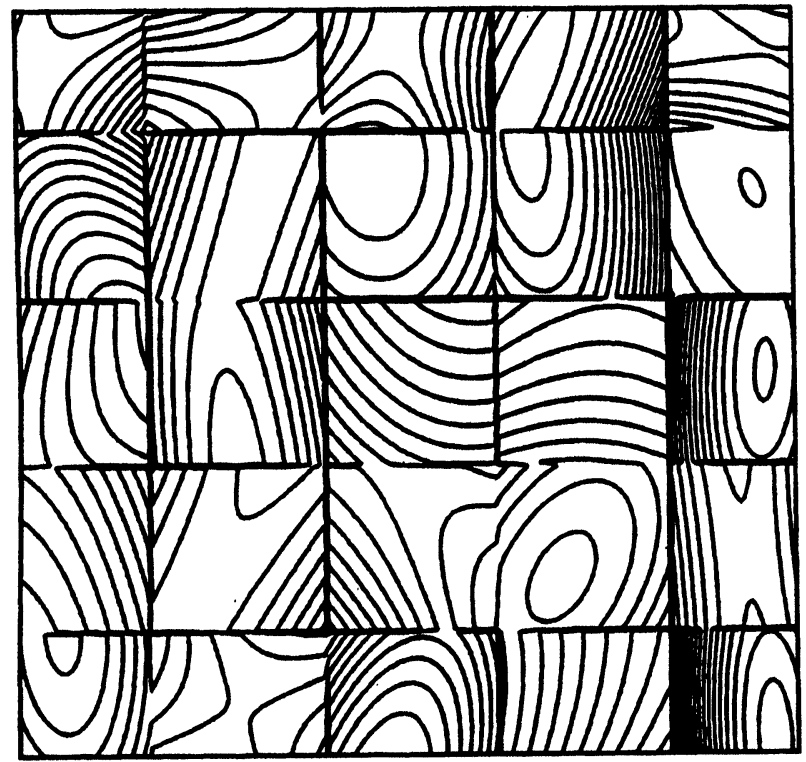

Figure 1 (a) Relative change of the specific volume $\delta V(r) / V_{0}$ in equidistant level lines inside a cluster consisting of cubic grains with different individual grain orientations. These orientations are determined by a random generator. The section represented in the figure is taken through the centre of the cluster $(r=0)$ along the plane $z=0$ containing the strain axis. Because of the large number of grains, we only display a narrow region centred at $r=0$.

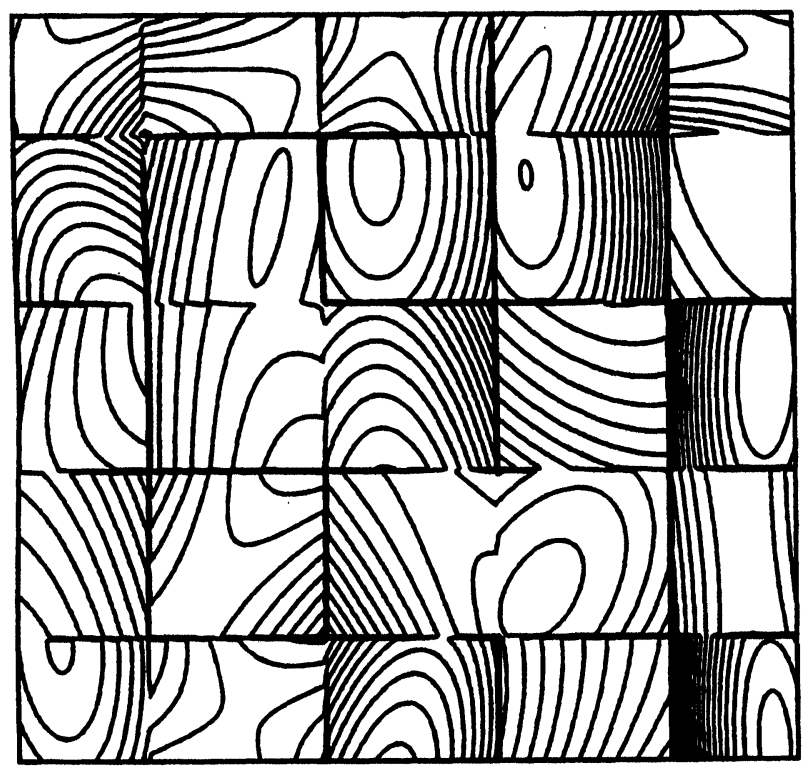

Figure 1 (b) The same field as in (a) for a second cluster which is identical to the first one except that we have taken a different orientation for the grain in the centre of the cluster. 


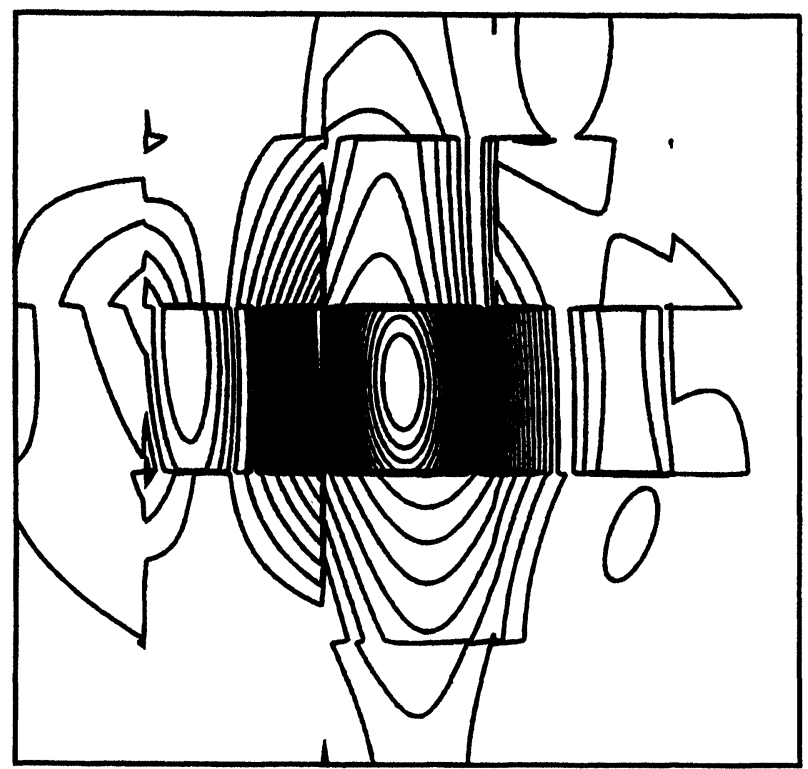

Figure 1 (c) Difference of the two fields displayed in (a) and (b). For clearness the displayed field is increased by a factor of 4 in comparison to (a) and (b).

also chosen at random. Figures 1 (a) and (b) show the relative change of the specific volume Eq. (1) inside a cluster 1 and a cluster 2, respectively. Since the boundary conditions are fulfilled for the stress the strain field is discontinuous at the grain boundaries which becomes apparent from Figures 1(a) and (b). Therefore discontinuities of elastic constants are mirrored in discontinuities of strain. The deformation of the central grain differs enormously between cluster 1 and 2 while for a grain which is in far distance from the origin the deformation is nearly the same in both clusters. The behaviour can be seen in more detail from Figure 1(c) where we have displayed the absolute value for the difference of the relative change of the specific volume of clusters 1 and 2. The difference attains its maximum in the central grain or its nearest neighbours and vanishes in the periphery of the section displayed in Figure 1(c). Since this decrease occurs far away from the surface of the whole cluster it is obvious that this is not due to surface effects of the cluster. Figures 2 and 3 show the respective behaviour of the two other grain shapes used for our investigations.

For a more quantitative description of the range of deformation fields we use the average of the relative change of the specific volume over all directions Eq. (2). Figures 4 to 6 show results for the three types of clusters corresponding to Figures 1 to 3 . Here, the absolute value of $\gamma(r)$ is the average over four clusters constructed for each grain shape, which only differ in the orientation of the central grain. $|\Delta \gamma(r)|$ is the respective average of the three difference fields between cluster 1 and clusters 2 to 4 , respectively. The relative change of the specific volume $|\gamma(r)|$ is, except for statistical variations, nearly constant over the entire region of the clusters. The steep decrease corresponds always to the "radius" of the cluster. In the case of a free surface the strain has to be identical to the given homogeneous strain at the surface. Therefore 

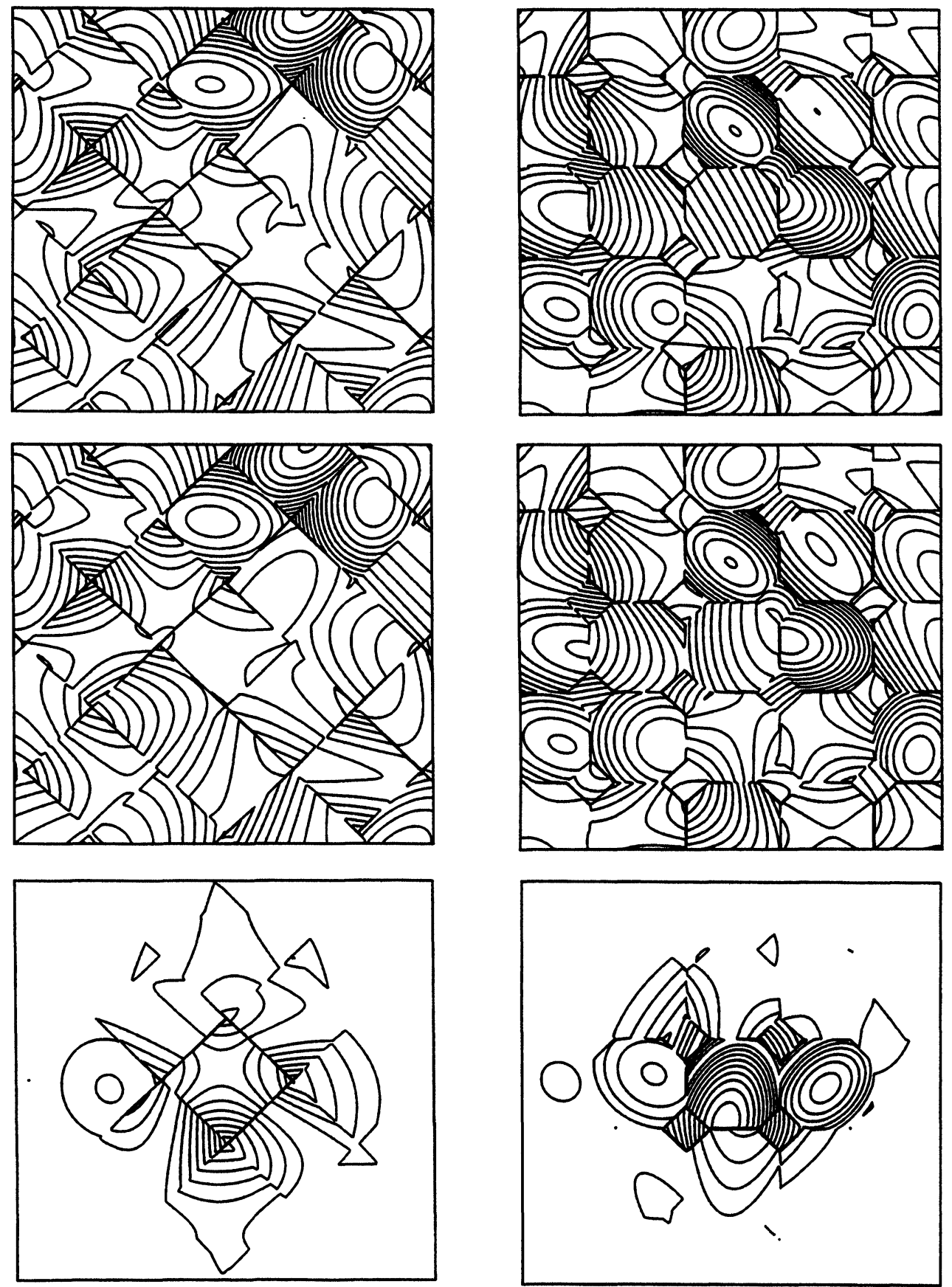

Figure 2 The same situation as in Figure 1 except that we have replaced the cubic grain shape by Wigner-Seitz cells of a face centred cubic lattice. The values of the level lines are the same as in Figure 1.

Figure 3 The same situation as in Figure 1 except that we have replaced the cubic grain shape by Wigner-Seitz cells of a body centred cubic lattice. The values of the level lines are the same as in Figure 1. 


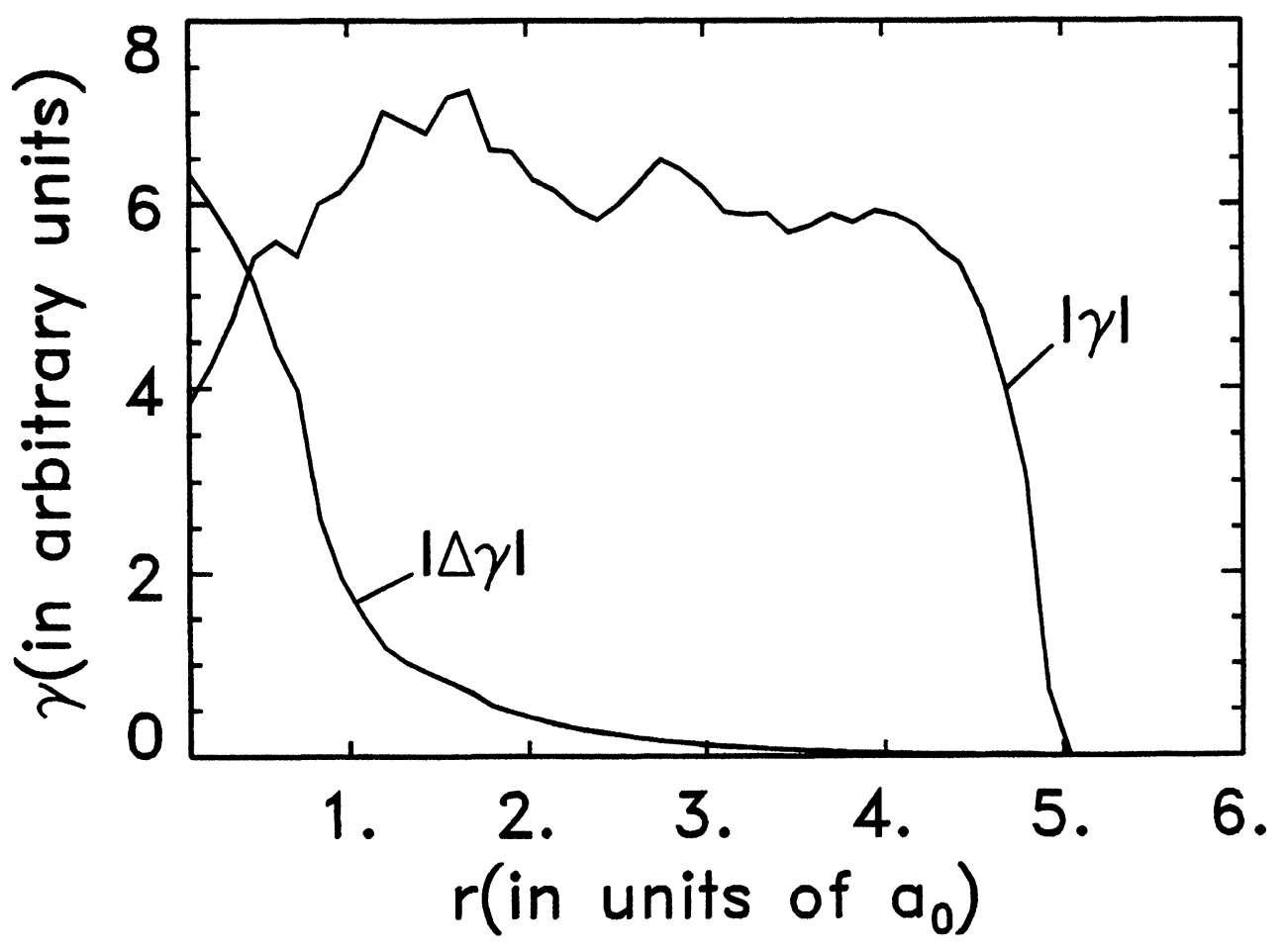

Figure 4 (a) Radial distribution of the relative change of the specific volume $|\gamma(r)|$ (see Eq. (2)) and $|\Delta \gamma(r)|$ averaged over 4 clusters consisting of cubes corresponding to Figure 1 . As in Figure 1 the 4 clusters are identical except for the orientation of the central grain. The parameter $a_{0}$ denotes the nearest-neighbours distance.

the relative change of the specific volume Eq. (1) would decrease monotonically to zero in the outer region of the cluster. Because of the embedding and the therefore combined boundary conditions this effect becomes negligible. Only for the cubic cluster (Figure 4(a)) a weak influence of the surface of the cluster can be seen. In far contrast, $|\Delta \gamma(r)|$ decreases enormously over the entire range of the cluster except for the small area of the central grain which lies inside a sphere of radius $a_{0} / 2$. To examine this behaviour in more detail we have additionally displayed $|\Delta \gamma(r)|$ in Figures 4 (b) to $6(\mathrm{~b})$ in a larger scale. As becomes evident from the different fits of rational functions, $|\Delta \gamma(r)|$ decreases in a manner between $1 / r^{3}$ and $1 / r^{4}$.

To investigate the influence of the grain shape on the range of misorientation effects in more detail we have extracted $|\Delta \gamma(\mathrm{r})|$ from Figures $4(\mathrm{~b})$ to $6(\mathrm{~b})$ (Figure 7). For the clusters consisting of cubes the range is a little bit larger than for the fcc- and bccclusters in which there is nearly an identical range. In the former case all angles inside the grains between different faces are right angles. In the latter case they are always larger. Stresses occuring near the vertices increase with decreasing size of angles. Therefore the additional stresses and as a consequence the resulting range due to a perturbation inside the sc-clusters are a little bit larger than for the two other types. 


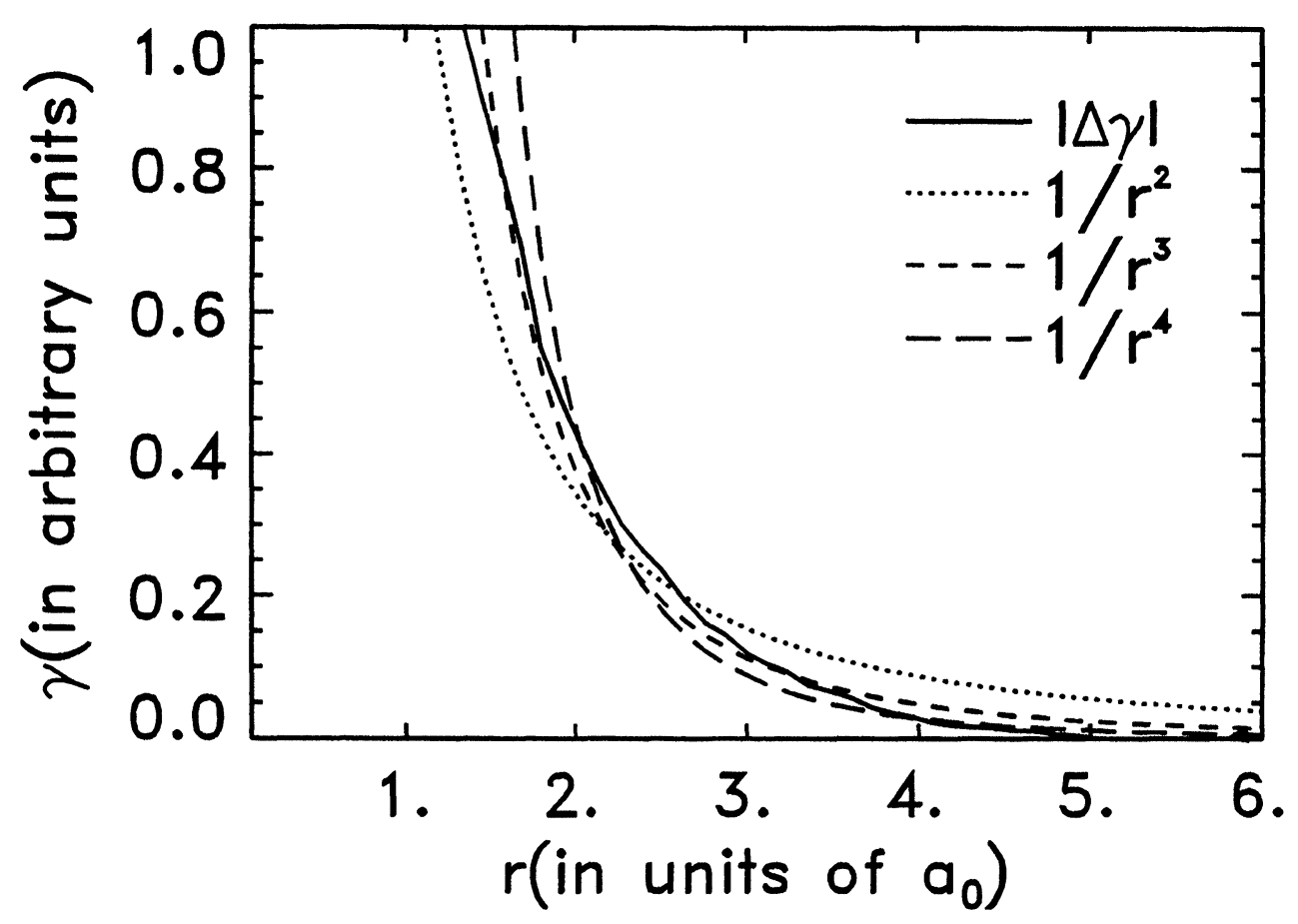

Figure 4 (b) Relative change of the specific volume $|\Delta \gamma(r)|$ as in (a). To fit different rational functions we have enlarged the scale. However, the arbitrary units remain the same as in (a).

An important further aspect is the relation between $|\Delta \gamma(r)|$ and the misorientation of the central grain. For these investigations we have used the third type of clusters which consist of bcc Wigner-Seitz cells. Again, macroscopically isotropic materials are simulated. The difference $|\Delta \gamma(r)|$ is taken from two clusters, which differ only in the orientation of the central grain. The first cluster is a kind of "reference" cluster. Its central grain has the orientation $g=\left(0^{\circ}, 0^{\circ}, 0^{\circ}\right)$ (Euler angles, see Bunge (1982)) with respect to the chosen coordinate system, the $\mathrm{x}$-axis of which is parallel to the direction of uniaxial strain. The orientation of the central grain in the second cluster was obtained by rotating $\alpha$ degrees around each of the three cubic axes. The results are shown in Figure 8 to 10 for different distances from the origin. For a rotation of the orientation around the axis parallel to the given uniaxial strain the elastic properties in the direction of deformation remain the same. For that reason the effect of this misorientation has to be much smaller than for a rotation perpendicular to this direction. This becomes apparent if one compares Figure 8 (rotation around the $x$-axis) with Figures 9 and 10 (rotation around the $y$ - or $z$-axis, respectively). The value at $\alpha=90^{\circ}$ is zero since the rotation axes coincide always with the 4-fold axes of the cubic symmetry. Therefore the values for $|\Delta \gamma(r)|$ increase monotonically up to $\alpha=45^{\circ}$ where they attain their maximum and then decrease monotonically up to $\alpha=90^{\circ}$. Because of statistical variations this behaviour is only approximately fulfilled. For example, the value for $\alpha=45^{\circ}$ in Figure 8 is obviously smaller than those for neighbouring orientations. 

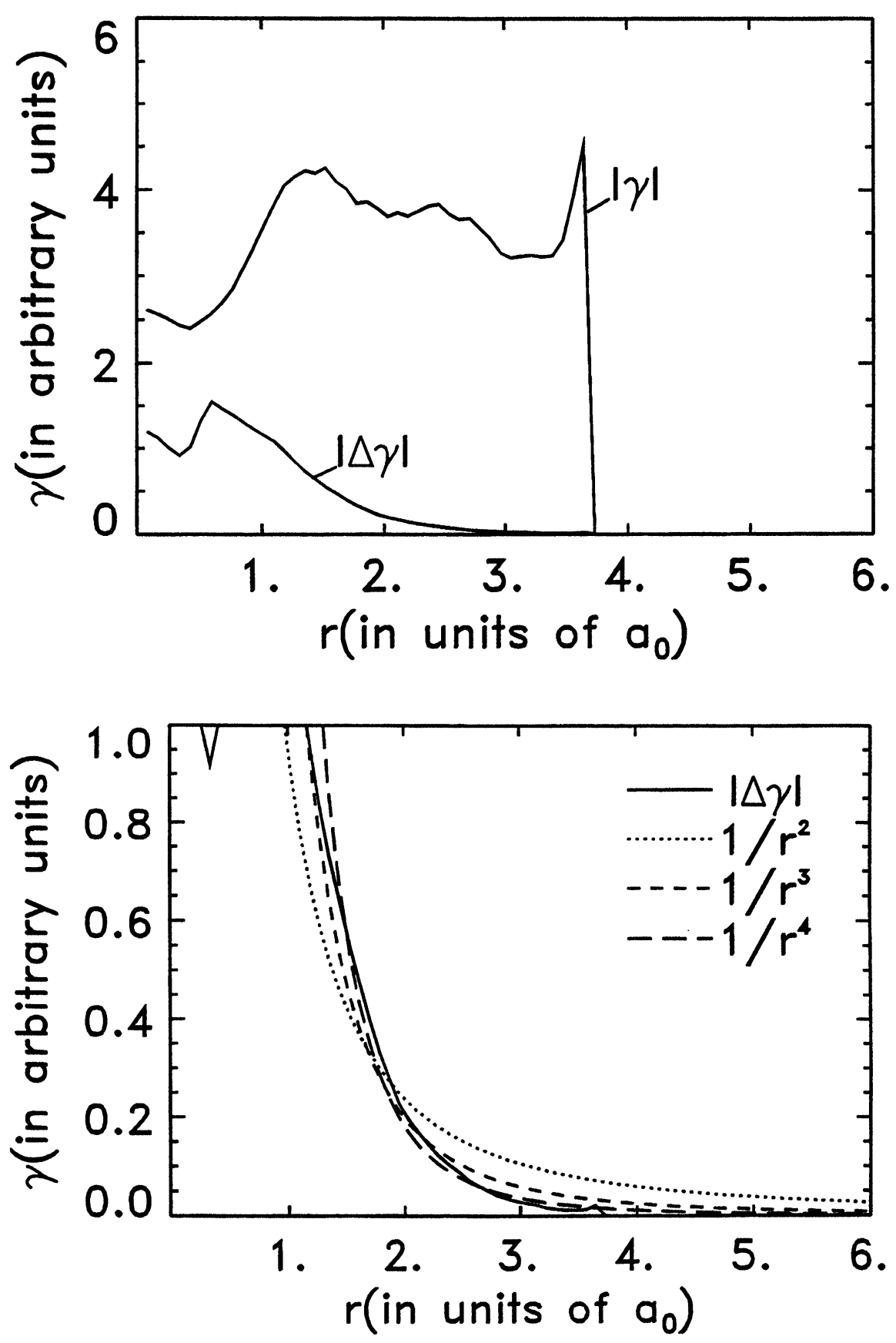

Figure 5 Course of $|\gamma(r)|$ and $|\Delta \gamma(\mathrm{r})|$, respectively, as in Figure 4 except that we have replaced the cubic grain shape by Wigner-Seitz cells of a face centred cubic lattice. This figure corresponds to Figure 2. The arbitrary units are the same as in Figure 4. The parameter $a_{0}$ denotes the nearest-neighbours distance. 

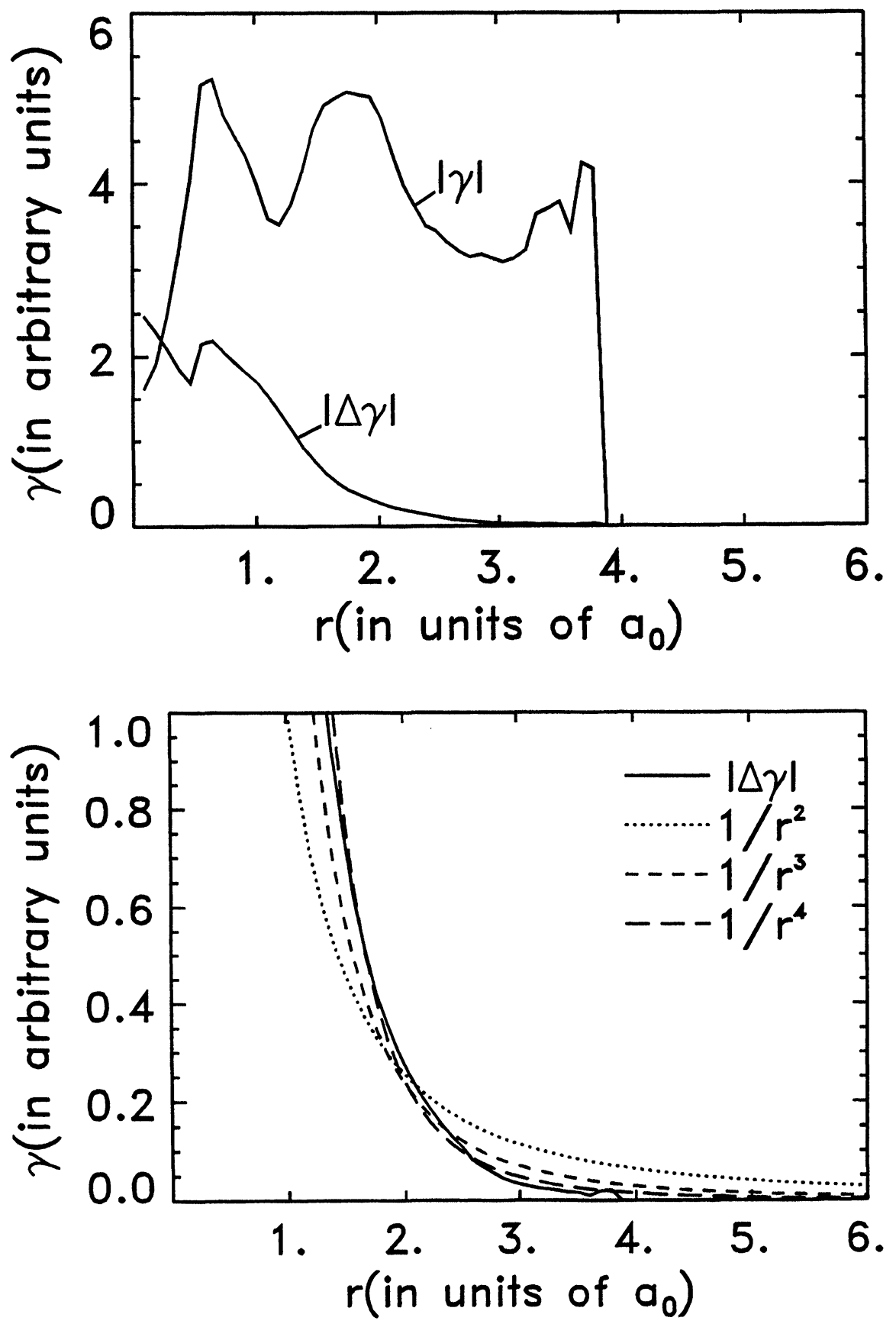

Figure 6 Course of $|\gamma(r)|$ and $|\Delta \gamma(r)|$, respectively, as in Figure 4 except that we have replaced the cubic grain shape by Wigner-Seitz cells of a body centred cubic lattice. This figure corresponds to Figure 3 . The arbitrary units are the same as in Figure 4 . The parameter $a_{0}$ denotes the nearest-neighbours distance. 


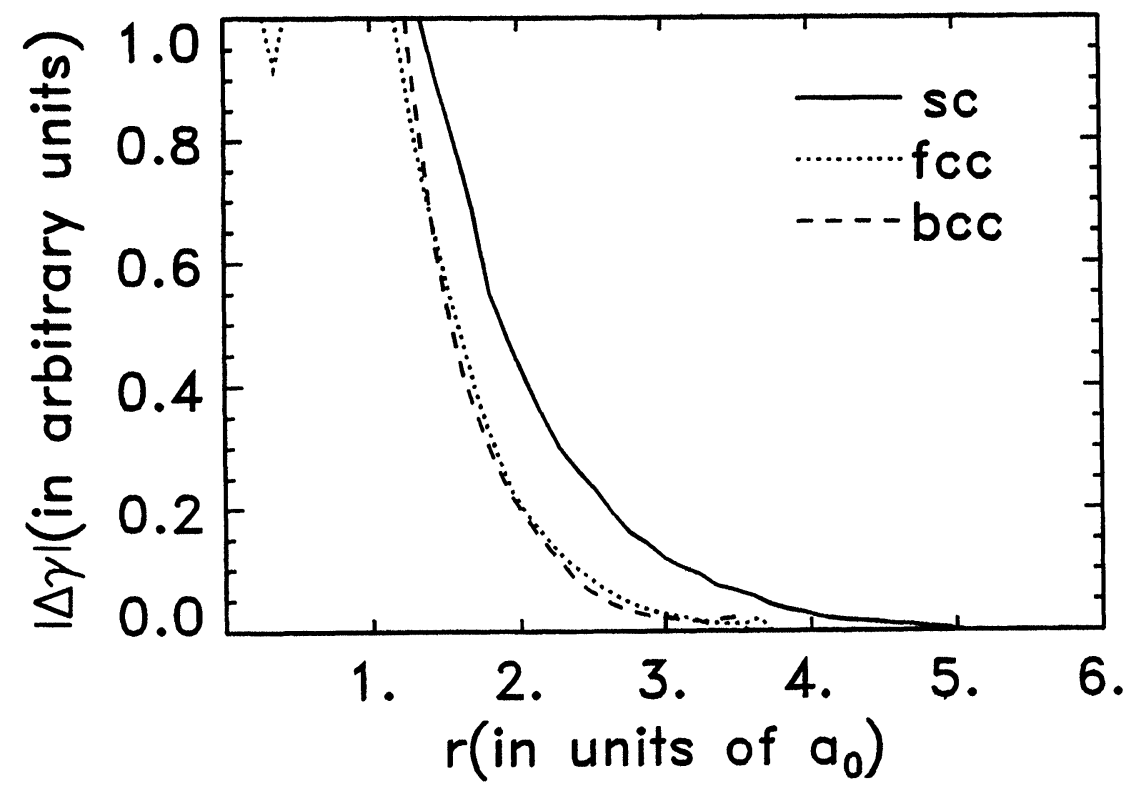

Figure 7 Course of $|\Delta \gamma(r)|$ for the three different grain shapes in comparison. The abbreviations stand for the respective Wigner-Seitz cells: simple cubic (sc), face centred cubic (fcc) and body centred cubic $(\mathrm{bcc})$. The curves are extracted from Figures $4(\mathrm{~b})$ to $6(\mathrm{~b})$. The arbitrary units are the same as in Figure 4. The parameter $a_{0}$ denotes the nearest-neighbours distance.

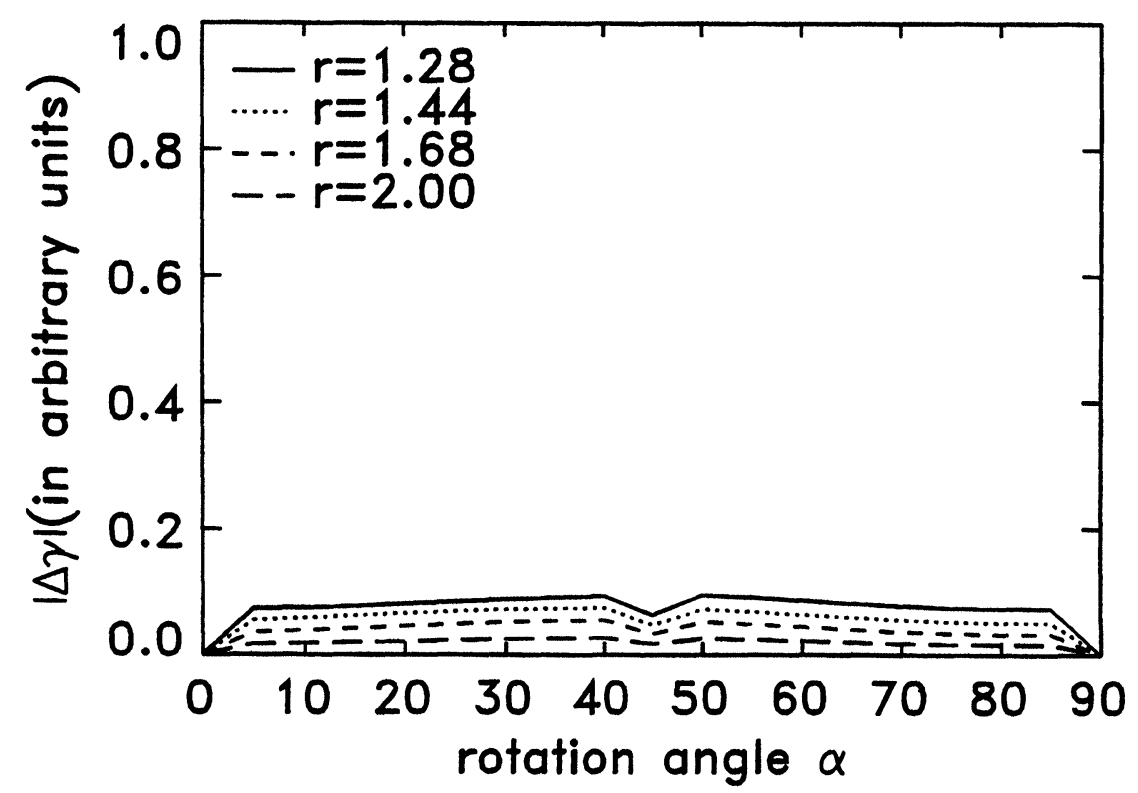

Figure 8 Values for $|\Delta \gamma(r)|$ as a function of the misorientation of the central grain. The relative change of the specific volume calculated for two clusters which only differ in the orientation of the central grain. In the first one the respective orientation is $\left(0^{\circ}, 0^{\circ}, 0^{\circ}\right)$. In the second cluster it was rotated through $\alpha$ degrees around the $x$-axis. The arbitrary units are the same as in Figure 4. 


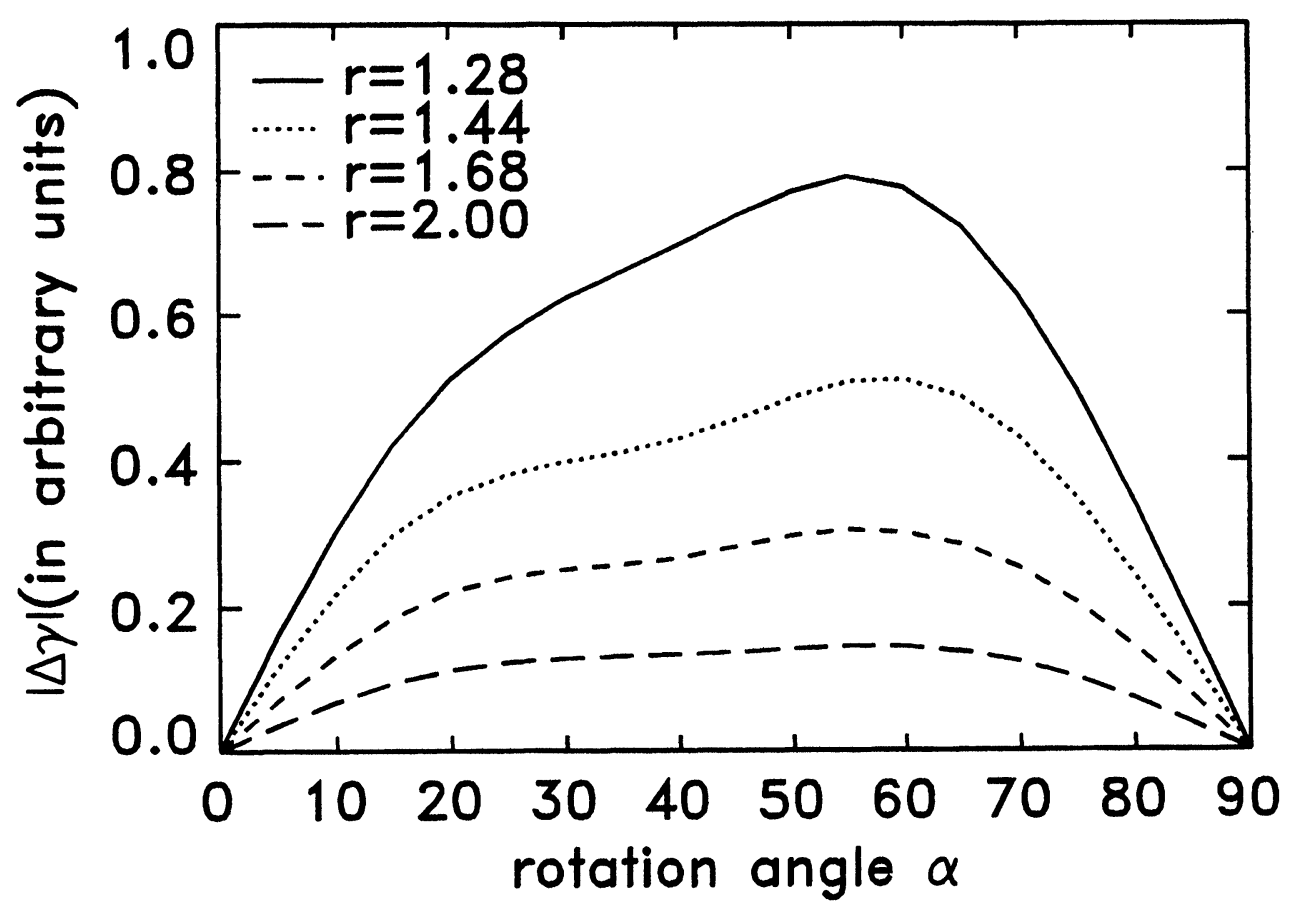

Figure 9 The same situation as in Figure 8 except that we have carried out the rotation around the $y$-axis. The arbitrary units are the same as in Figure 4.

\section{CONCLUSIONS}

In this paper the strain field arising from discontinuities of the elastic constants across grain boundaries was studied. The discontinuities are due to different crystal orientations in neighbouring grains. The distance to which the influence of one particular grain reaches can be estimated by changing the orientation of this grain while leaving the orientations of all others constant. This leads to an additional elastic field $\Delta \gamma$ in the grain itself and in its vicinity. In far distance from the grain the additional deformation decreases stronger than $1 / r^{3}$. For that reason the deformation field is negligible in a distance of more than two or three grain diameters. As a consequence of that, correlation effects of only nearest and next-nearest neighbours have to be taken into account, in addition to the texture, in order to obtain more reliable results for the effective elastic properties than those of the Voigt and Reuss averages.

The present results also confirm once more - as was already tested earlier by other methods - that the chosen sizes of the clusters i.e. 365, 201 and 181 grains respectively, are big enough for the estimation of grain shape and orientation correlation effects.

The results show that the shape of the grains influences the strain field. The highest value of $\Delta \gamma$ inside the central grain is observed in the case of cubic grains. In the other two grain shapes i.e. dodecahedron and cubo-octahedrons this value is much 


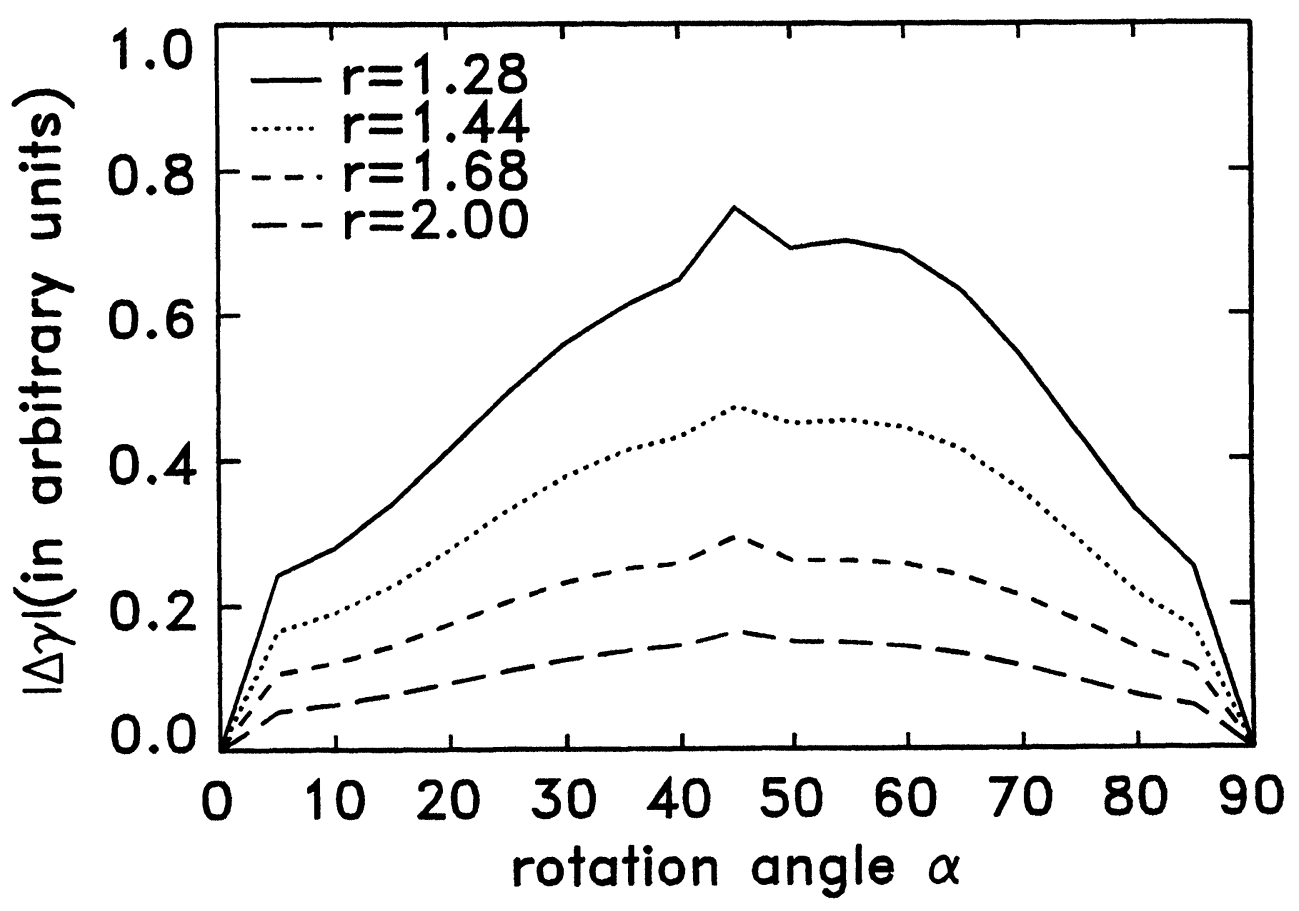

Figure 10 The same situation as in Figure 8 except that we have carried out the rotation around the $z$-axis. The arbitrary units are the same as in Figure 4.

smaller. The same tendency is also seen in the decreasing field in the vicinity of the central grain. This effect is in accordance with earlier results (Kiewel, Bunge and Fritsche (1995)) for the effective elastic constants which also depend in a similar way on grain shape.

The additional strains must increase with the absolute values of the discontinuity of the elastic properties across the grain boundaries. These, in turn, increase with increasing misorientation. Figures 8 to 10 illustrate the influence of different tensor components on the strain field through their variation with misorientation. Figure 8 , for instance, can be understood in terms of varying Poisson ratio and its interaction with side-neighbours even with constant Young's modulus in the direction of the applied tensile strain.

It is also seen that even small angle boundaries in the order of $5^{\circ}$ have a clearly visible effect on the strain field and hence on the effective elastic constants.

It may be allowed to understand the strain fields Figures 1 to $3 a, b$ as super-positions of contributions from all individual grains i.e. superpositions of fields of the kind of Figures 1 to $3 \mathrm{c}$ the amplitudes of which vary with misorientation according to Figures 8 to 10 . Hence, the superimposed fields will depend on the misorientation distribution function MODF as will be demonstrated elsewhere. 


\section{Acknowledgments}

The present work was financially supported by the Deutsche Forschungsgemeinschaft which is gratefully acknowledged.

\section{References}

Bunge, H. J. (1982). Texture Analysis in Materials Science, Butterworths, London.

Hashin, Z. and Shtrikman, S. (1962a). On some variational principles in anisotropic and nonhomogeneous elasticity, J. Mech. Phys. Solids, 10, 335.

Hashin, Z. and Shtrikman, S. (1962b). A variational approach to the theory of the elastic behaviour of polycrystals, J. Mech. Phys. Solids, 10, 343.

Hill, R. (1952). The elastic behaviour of a crystalline aggregate, Proc. Phys. Soc. A 65, 351.

Kiewel, H. and Fritsche, L. (1994). Calculation of effective elastic moduli of poly-crystalline materials including non textured samples and fiber textures, Phys. Rev. B 50, 5.

Kiewel, H., Bunge, H. J. and Fritsche, L. (1995). Effect of the grain shape on the elastic constants of polycrystalline materials, Textures and Microstructures, in press.

Kröner, E. (1977). Bounds for effective elastic moduli of disordered materials, J. Mech. Phys. Solids, 25, 137.

Reuss, A. (1929). Berechnung der Flie $\beta$ grenze von Mischkristallen auf Grund der Plastizitätsbedingung für Einkristalle, Z. Angew. Math. Mech., 9, 49.

Voigt, W. (1910). Lehrbuch der Kristallphysik, Teubner, Berlin.

Zeller, R. and Dederichs, P. H. (1973). Elastic Constants of Polycrystals, phys. stat. sol., (b) 55, 831. 